\title{
Estimation of small woodlot and tree row attributes in large-scale forest inventories
}

\author{
F. Baffetta - L. Fattorini · P. Corona
}

Received: 10 April 2009 / Revised: 23 September 2009 / Published online: 15 October 2009

(C) Springer Science+Business Media, LLC 2009

\begin{abstract}
Forest surveys performed over a large scale (e.g. national inventories) involve several phases of sampling. The first phase is usually performed by means of a systematic search of the study region, in which the region is partitioned into regular polygons of the same size and points are randomly or systematically selected, one per polygon. In most cases, first-phase points are selected and recognized in orthophotos or very high resolution satellite images available for the whole study area. Disregarding the subsequent phases, the first phase of sampling can be effectively adopted to select small woodlots and tree rows, in the sense that a unit is selected when at least one firstphase point falls within it. On the basis of such a scheme of sampling, approximately unbiased estimators of abundance, coverage and other physical attributes readily measurable from orthophotos (e.g. tree-row length) are proposed, together with estimators of the corresponding variances. A simulation study is performed in order to check the performance of the estimators under several distributions of units over the study area (random, clustered, spatially trended).
\end{abstract}

Keywords Tessellation stratified sampling · Hansen-Hurvitz estimator · Horvitz-Thompson estimator · Variance estimator · Monte Carlo study · Trees-outside-forests

\footnotetext{
F. Baffetta $\cdot$ L. Fattorini $(\varangle)$

Dipartimento di Metodi Quantitativi, Università di Siena,

P.za S. Francesco 8, 53100 Siena, Italy

e-mail: fattorini@unisi.it

P. Corona

Dipartimento di Scienze dell'Ambiente Forestale e delle sue Risorse (DISAFRI),

Università della Tuscia, via San Camillo de Lellis, 01100 Viterbo, Italy
} 